\title{
Managing Deliberate Self Harm patients: Knowledge and practices among hospital doctors in Galle district
}

\author{
Chandrasiri $\mathrm{A}^{1}$, Gunethunga $\mathrm{W}^{2}$, Rajapaksha $\mathrm{H}^{3}$
}

\begin{abstract}
Introduction

Deliberate Self-Harm (DSH) has emerged as an important health issue in Sri Lanka. It is one of the strongest risk factors for completed suicides.

\section{Methods:}

A questionnaire based descriptive cross-sectional study with a qualitative component was done in three hospitals in Galle district, in Southern Sri Lanka. Selection of variables was done by reviewing selected literature and curricula of basic training of medical officers. A series of in depth interviews were conducted to supplement the findings by using purposive sampling. Multivariate analysis was done to determine factors associated with satisfactory level of performances. Qualitative data analysis was done for in depth interviews.
\end{abstract}

\section{Results:}

Overall knowledge and practices were shown to be satisfactory but deficiencies were noted in certain aspects. Male gender (OR 3.560) and having a work experience of $<5$ years (OR 6.184) showed significantly higher level of knowledge while age $<30$ years (OR 4.497) showed satisfactory level of practices. Lack of priority given for psychiatric care stood as the main barrier in delivering satisfactory care. Excess work load, lack of competency and inadequate training appeared to be contributory factors.

\section{Conclusions:}

More training opportunities on psychiatric management DSH patients should be included. Competencies on preliminary risk assessments and providing basic psychological support should be improved.

Key words: Deliberate self harm, Medical knowledge, Medical practice, Guidelines, Sri Lanka

\section{Introduction}

Deliberate Self-Harm has emerged as an important health and a social issue worldwide. It is one of the strongest

1. Senior Registrar in Community Medicine, Ministry of Health, Colombo, Sri Lanka,

2, Professor in Community Medicine, Faculty of Medicine, University of Colombo, Sri Lanka

3. Senior Lecturer, Faculty of Medicine, University of Ruhuna, Sri Lanka

Corresponding author - Amila Chandrasiri, email: amilachan@yahoo.com

(iD) https://orcid.org/0000-0002-3242-1489 risk factors for completed suicides and also provides an opportunity to prevent it. Though we do not have exact figures, it is estimated that each year nearly 30000 people engage in deliberate self harm (DSH) in Sri Lanka, as 10 to 20 times the number who complete suicide self harm. ${ }^{1}$ The annual death toll due to completed suicides has ranged around 3000 over past few years according to Department of Police data.

An episode of intentional self-harm that did not lead to death and irrespective of intention to die is considered as an act of DSH. ${ }^{2}$ Since DSH is the most significant predictor of subsequent completed suicides, delivering satisfactory psychiatric care to these individuals is of importance. In the hospitals DSH patients are usually managed in general medical and surgical wards. As only a proportion of patients are seen by psychiatry team due to ward

The articles in this journal are licensed under a Creative Commons Attribution 4.0 International License. 
referral practices, ${ }^{3,4}$ doctors who work in general wards have a crucial responsibility in managing DSH.

Competency of hospital staff is identified as an important determinant of quality of service delivered. In improving hospital management practices of a particular aspect of care, assessing the current status of knowledge and practices among staff should be the starting point. ${ }^{5}$ This study intended to assess the status of knowledge and practices among doctors in delivering psychiatric care to patients admitted following DSH in District of Galle.

\section{Methodology}

A questionnaire based descriptive cross-sectional study with a qualitative component was conducted in July 2015. The Study was carried out in medical and surgical wards of three hospitals in Galle district, Southern Sri Lanka. The three hospitals, Teaching Hospital - Karapitiya, Base Hospital - Balapitiya and Base Hospital - Elpitiya, drain more than $80 \%$ of the population of district. ${ }^{6}$

The study population consisted 194 medical staff namely; consultants, post graduate trainees and other medical officers, later group including interns. There was no sampling done as all the eligible individuals in the study population were invited for the study.

Selection of variables for the questionnaire was done after reviewing selected international guidelines on management of DSH patients in hospital setting including National Institute of Clinical Excellence (NICE) and Australian National Guidelines. Recommended Text books in psychiatry for undergraduates and curricula of basic undergraduate training of medical officers were also reviewed. Main aspects of management of patients admitted following DSH were identified by reviewing these guidelines and text books. Expected level of knowledge and practices were defined by going through above mentioned curricula. Draft of the questionnaire was given to an expert panel consisting of a consultant psychiatrist and two consultant physicians for their comments. Face validity, content validity and consensual validity were assessed by obtaining comments of the panel. It was pretested and relevant modifications were done to improve the clarity and acceptability of questionnaires. All questions had either multiple choice or dichotomous responses. There were eight knowledge and eight practice related items in the questionnaire.

Principal investigator (AC) himself delivered the questionnaires and was present throughout to answer any queries. Upon receiving the questionnaires participants were instructed not to discuss the questions among themselves. Maximum of three attempts were made for those who couldn't contact. Eligible population was reached over a period of two weeks. A series of in depth interviews were conducted to supplement the descriptive study. Sample was identified to represent different categories of doctors and individuals were identified by purposive sampling. An interviewer guide which was in semi structured format was followed. It included selected aspects of psychiatric management of DSH which were qualitative in nature.

Relevant descriptive statistics were calculated. Multivariate analysis was done to determine factors associated with satisfactory level of performances. For in depth interviews ongoing analysis was done. Themes were identified as they emerged during interviews. Sorting, organizing and indexing were done. Common themes noted and explanations were given. Minimum disruption to routine patient care was assured. Ethical approval for the study was obtained from Ethics review committee of Faculty of Medicine, Karapitiya.

\section{Results}

Table 1: Characteristics of participants

\begin{tabular}{|c|c|c|}
\hline \multirow[b]{2}{*}{ Characteristic } & \multicolumn{2}{|c|}{ Participants } \\
\hline & $\begin{array}{c}\text { Frequency } \\
(n=118)\end{array}$ & $\begin{array}{c}\text { Percentage } \\
(100.0 \%)\end{array}$ \\
\hline \multicolumn{3}{|l|}{ Age category (years) } \\
\hline Below 30 & 73 & 61.9 \\
\hline 30 and above & 45 & 28.1 \\
\hline \multicolumn{3}{|l|}{ Gender } \\
\hline Male & 61 & 51.7 \\
\hline Female & 57 & 48.3 \\
\hline \multicolumn{3}{|l|}{$\begin{array}{l}\text { Graduated Medical } \\
\text { faculty }\end{array}$} \\
\hline Colombo & 40 & 33.9 \\
\hline Karapitiya & 44 & 37.3 \\
\hline Peradeniya & 16 & 13.6 \\
\hline Other & 18 & 15.2 \\
\hline \multicolumn{3}{|l|}{$\begin{array}{l}\text { Had psychiatry as a } \\
\text { separate subject in }\end{array}$} \\
\hline \multicolumn{3}{|l|}{ MBBS course } \\
\hline Yes & 85 & 72.0 \\
\hline No & 29 & 24.6 \\
\hline Can't remember & 2 & 1.7 \\
\hline Missing & 2 & 1.7 \\
\hline \multicolumn{3}{|l|}{$\begin{array}{l}\text { Work Experience } \\
\text { (years) }\end{array}$} \\
\hline 5 and below & 86 & 72.9 \\
\hline 6 and above & 32 & 27.1 \\
\hline \multicolumn{3}{|l|}{$\begin{array}{l}\text { Current working } \\
\text { station }\end{array}$} \\
\hline TH Karapitiya & 85 & 72.0 \\
\hline BH Balapitiya & 21 & 17.8 \\
\hline BH Elpitiya & 12 & 10.2 \\
\hline
\end{tabular}


Out of 142 medical staff available in all three study settings, 118 duly filled questionnaires were obtained with a response rate of $83.1 \%$. Sample consisted of 13 Consultants (11.0\%), 5 Senior Registrars (4.2\%), 13 Registrars (11.0\%), 30 Senior House Officers (25.4\%) and 57 Intern House Officers (48.3\%). Observed demographic and other characteristics related to professional and training statuses are presented in table 1.

Table 1: Characteristics of participants The Knowledge component consisted of 8 items and proportions of correct responses for each item is presented in figure 1.

Figure 1: Performance for knowledge component of the questionnaire

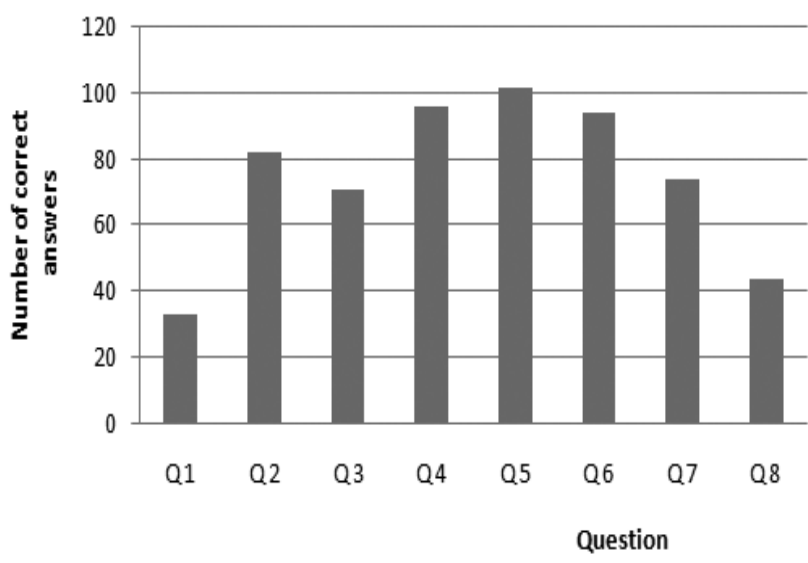

Q 1 - Whether all the DSH cases have a higher risk of suicides( Answer: Yes), Q 2 - Whether most of them are having psychiatric disorders( Answer: No), Q 3 Whether delivering psychiatric care should begin at the point of admission (Answer: Yes), Q 4 -Whether those used less lethal methods do not need psychiatric assessment ( Answer: No), Q 5 - Whether even impulsive acts need psychiatric assessment (Answer: Yes), Q 6 - Whether if there are no ongoing suicidal ideas there is no need of further assessment (Answer: No), Q 7 - Whether asking about suicidal intention increases the risk of future suicides( Answer: No), and Q 8 - Whether all DSH cases should be referred to a psychiatrist( Answer: No).

Only 33 (28.0\%) of the study population agreed that all patients with DSH carry a higher risk of suicides than normal, but 82 (69.5\%) agreed that most of DSH cases don't have psychiatric disorders and 96 (81.4\%) agreed that even patients who used less lethal methods should undergo psychiatric assessment. A large majority of the study population ( $n=102,86.4 \%$ ) agreed that even impulsive DSH acts deserve psychiatric assessment in ward and again most of the study population ( $n=94$, $79.7 \%$ ) agreed that even there is no ongoing suicidal ideation they should undergo psychiatric assessment. There were $71(60.2 \%)$ who mentioned that provision

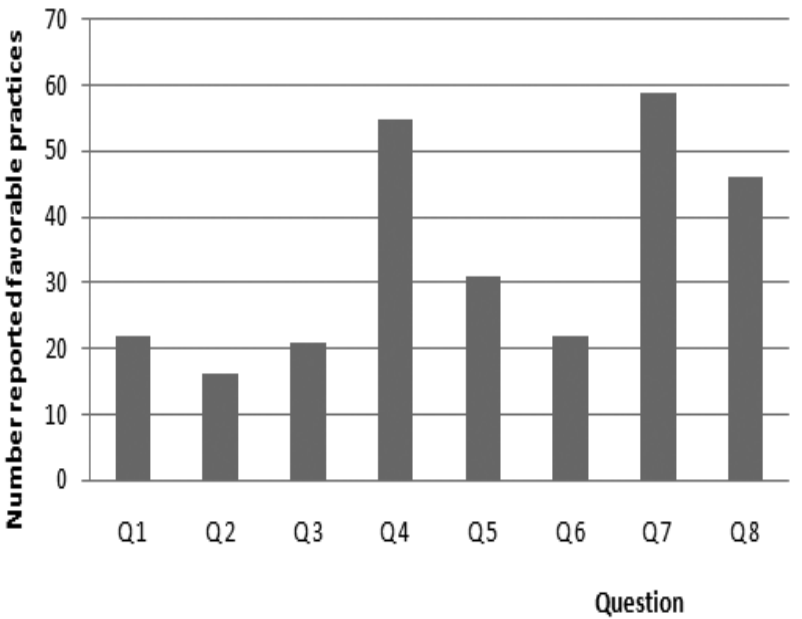

of psychiatric care should begin from the point of admission, however only $74(62.7 \%)$ correctly pointed out that asking about suicidal intention from self-harm patients does not increases the risk of future suicides.

But only small proportion ( $n=44,37.3 \%$ ) mentioned that every DSH case do not need psychiatric referral. Similarly, clinical practice was assessed by administering 8 items. Here the response given as "Do always" was considered as the ideal practice. Distribution of ideal status of practices is shown in figure 2 .

Q 1 - Whether had a training on managing DSH patient at ward setting after passing out from medical school, Q 2 - Whether a suicidal risk assessment was done before referring them to psychiatric unit, Q 3 - Whether obtaining a brief psychiatric history from patients was practiced, Q 4 - Whether inquired about past attempts of self harm from patients, Q 5 - Whether speaking with family members were done in history taking, Q 6 - Whether involved family members in preparing the management plan, $Q 7$ - Whether explaining the importance of psychiatric referral and follow up to family members and Q 8 Whether a double check was done to see if suicidal risk assessment and psychiatric referral carried out. Approximately one fifth of study population $(n=22$, $18.6 \%$ ) acknowledged that they have had a training in managing a DSH patient after passing out from medical faculty. Only a minority $(n=16,13.6 \%)$ were doing a suicidal risk assessment for every patients with DSH before referring them to psychiatric unit and only 21 $(17.8 \%)$ reported that they obtain detailed psychiatric history from every DSH patient. However 55 (46.6\%) subjects inquired about past attempts of self-harm from all patients with DSH. Involving family members in managing patients with DSH appeared to be as low as $31(26.3 \%)$ mentioned that they speak with family members of patients while obtaining the history and only 22 (18.6\%) acknowledged that they involve family members in preparing the management plan. 
Table 2: Variables in the Logistic Regression Model for the Identification of Associations With Satisfactory Level of Knowledge and Practices Among Doctors

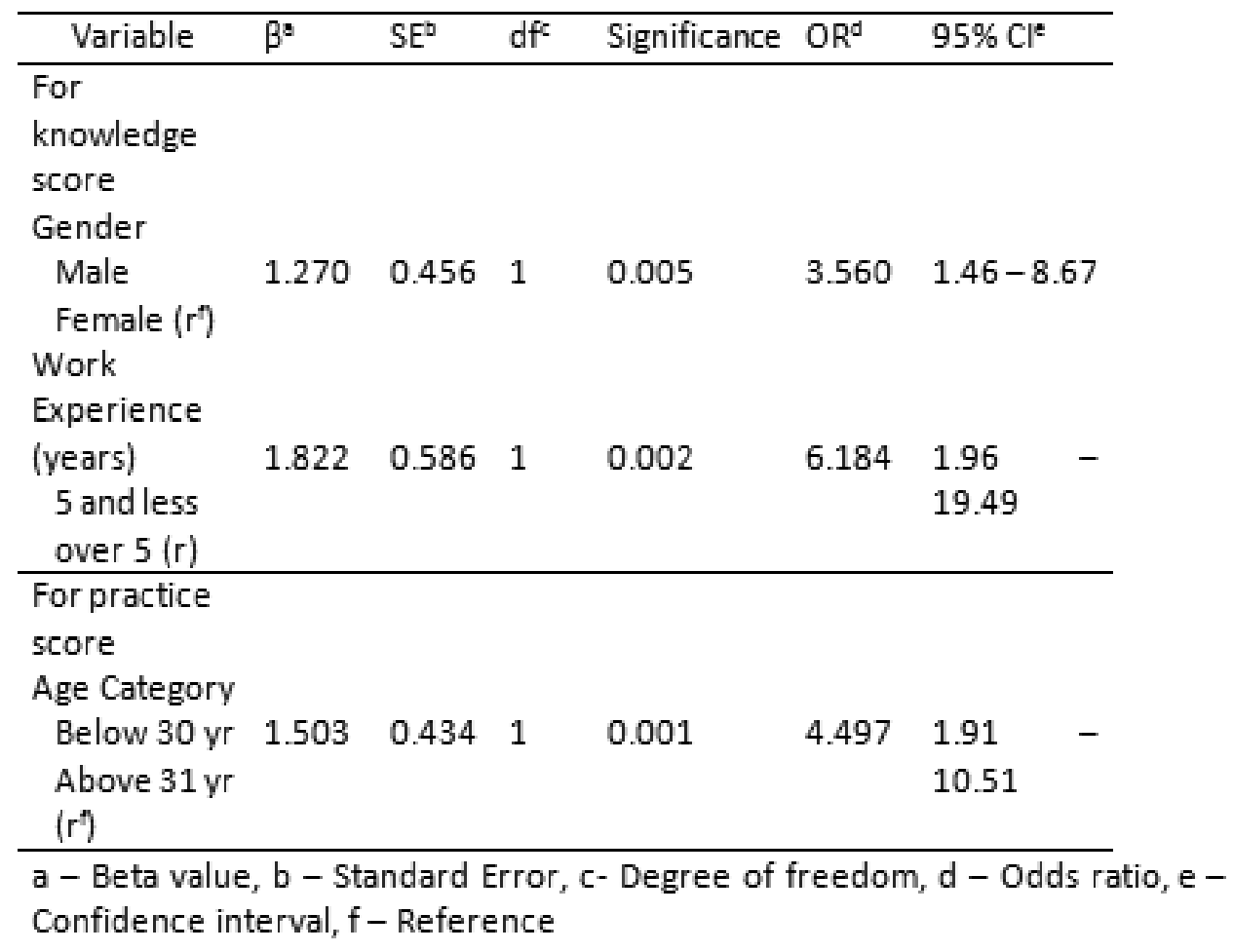

For the purpose of further analysis, overall scores were allocated to knowledge and practices components. Mean knowledge score among entire study population was 23.95 (maximum 40) with SD of 5.83 while mean practices score was 26.26 (maximum 40 and SD 4.73). To elicit association of level of knowledge and practices with the selected variables, multivariate analysis was done. For the purpose of analysis knowledge and practices scores were categorized into satisfactory and unsatisfactory levels. Cut off marks were determined after obtaining expert opinion.

Level of knowledge was taken as the dependent variable (having satisfactory level of knowledge was coded as 1 while having unsatisfactory level was taken as 0 ). Then variables were selected for logistic regression. Only two variables were found to be significantly associated with satisfactory level of knowledge and they were chosen for further analysis. Variables associated with satisfactory level of practices were assessed in the similar manner and only age category was noted to have significant associations.

Among participants male gender (OR $3.560,95 \% \mathrm{Cl}$ $1.46-8.67)$ and those who has had a work experience of 5 years or less in General ward setting (OR: 6.184, $95 \% \mathrm{Cl} 1.96-19.49$ ) showed significantly higher level of knowledge. When age was considered, those who were below 30 years of age (OR 4.497, $95 \% \mathrm{Cl} 1.91$
- 10.51) showed satisfactory level of practices, compared to those who were older.

For the qualitative component sample consisted of one consultant physician, one consultant surgeon, two senior registrars, four registrars, three senior house officers (SHO's) and four intern house officers (IHO's) from both medical and surgical units.

When asking about practice of performing suicidal risk assessment and obtaining psychiatric history, SHO B said "We just ask whether you did it with the intention of dying. We just ask it for our knowledge" Surgeon A said "We don't assess the suicidal risk or psycho-social status. We refer every DSH case to psychiatry unit"

While exploring the reasons for above practices, Surgeon A said "We get nearly 150 patients during a casualty day. My staff has no time other than attending to the surgical care of those patients."

Medical SR B said "During busy casualty days we prioritize patients. We need to attend urgent medical cases. So we may not give much attention for selfharm patients."

Excess work load and lack of priority were elicited as main factors for not paying due attention for preliminary psychiatric assessments. Assuming that, it's a duty considered vested with psychiatry unit and self 
perceived lack of competency were other themes elicited.

Medical SR B said "Anyway psychiatric team will assess and do the needful. So why we should bother."

Surgical Registrar C said "We do the assessment of suicidal risk. But we don't know whether we are doing it in proper way. Honestly I don't remember."

When asking about referring practices to psychiatry unit; mixed responses were given:

Physician A said "We usually refer all the patients to psychiatry unit if admitted following self-harm."

IHO B said "I think most of them have undetected psychiatric illnesses. So it's better to refer all of them"

Another Registrar (A) mentioned "Not to take any risk we refer every DSH patient to psychiatry unit" though it wasn't mention directly, avoidance of risk or blame appeared to be the thinking behind.

Registrar C mentioned "If the patient is apparent to be having a psychiatric illness we definitely refer."

Physician A said, "If there is a serious risk of suicide we refer."

Physician A further described how she decide on referral, "We don't do according to a scale. I usually do it according to my clinical experience, considering the nature of problem. I also ask about the history of past attempts."

Describing the psychiatric referral practices for DSH patients, participants noted that some medical and surgical units used to refer each and every case of DSH irrespective of any other factor and some units refer patients on individual basis considering suicidal risk and presence of psychiatric symptoms. But in certain units there was no coherent reasoning. Referrals appeared to be done haphazardly. However in almost all the units the decision on referral was made by the most senior clinician, either Consultant or Senior Registrar. It was found that there were no guidelines to determine who should refer and who should not and individual practices of consultants determined the referral practices. But most of participants pointed out the importance of such guidelines.

\section{Discussion}

Both mean knowledge and practice scores were above $50 \%$. But knowledge was deficient in certain aspects and a similar pattern was noted regarding practices as well. Most importantly medical officers not realizing DSH as an event that increases the future risk of suicides needs attention. Not linking an act of self harm to an expression of suicidal ideation was evident and this may result in not paying due attention to the mental health needs and attending to medical problems only . Though knowledge seems to be satisfactory, transforming that into practice appears to be poor in certain aspects. Specially performing suicidal risk assessment before referring them to psychiatric unit and obtaining a brief psychiatric history from patients were poorly practiced.

In multivariate analysis male gender (OR 3.56) and having work experience of less than 5 years (OR 6.18) were significantly associated with satisfactory level of knowledge. Similarly below 30 years of age category was significantly associated with satisfactory level of practices with OR of 4.50. Fresh knowledge and enthusiasm by younger medical staff could be one explanation. Recent curriculum changes emphasizing psychiatry could also have played a role.

Several reasons emerged for not performing suicidal risk assessment before referring. Excess work load vested on medical staff during admission days (which is also known as casualty days) was mentioned as a dominant reason. According to hospital regulations each day of the week is allocated to a particular medical or surgical ward and all the patients presented during that day are diverted to those wards. This results in heavy patient influx, reducing the available time for the initial assessment. Assumption that psychiatry team will do the needful was the other important factor for non performance of a psychiatric assessment. It further reiterated the practice of compartmentalization of provision of medical and psychiatric care for these patients. Other common theme was lack of competency and this finding indicates the need to incorporate relevant knowledge and skills to the basic training of doctors. Several studies had shown that initial suicidal risk assessment links with prolong treatment success 7 and every DSH patient should undergo risk assessment at the earliest time possible. ${ }^{8}$

It was apparent that in the units where all the DSH patients were referred for psychiatric assessment, consultants advised the whole staff to do the same. Believing that all DSH patients have psychiatric illnesses, thinking that it's an essential component of management and avoiding risk were apparent factors for universal referral. But during the quantitative assessment of knowledge among doctors, it was shown that most think that majority of DSH patients do not have psychiatric illnesses. Some doctors also think, psychiatric referral is essential for the completion of management. But most of doctors pointed out the importance of the availability of a guideline.

\section{Conclusion and recommendations}

More training opportunities on psychiatric management of patients admitted with DSH should be included into curricula of basic training of doctors. Competencies on preliminary risk assessments and provision of basic psychological support should be improved 
through this training. We recommended reviewing current curricula of MBBS courses in all the medical schools to find ways to modify and include relevant missing content. When conducting training programs, female doctors and senior medical officers (who have a work experience more than 5 years) require priority. We recommend guidelines on psychiatric assessment and referral of DSH patients to be introduced to General medical and surgical wards of all hospital where these patients are being treated.

\section{References}

1.Lonnqvist J. Epidemiology and causes of suicide. in Oxford Text Book of Psychiatry. Oxford: Oxford University Press; 2009.

2.Gelder M, Harrison, P. and Cowen, P., editors. Shorter Oxford Textbook of Psychiatry. 6th ed: Oxford Universiry Press; 2006.

3.Chandrasiri A, Gunethunga W, Rajapakse H. Improving Psychiatric Management and Community Follow Up of Persons Who are Admitted With Deliberate Self Harm to Selected Health Institutions in Galle District: Post Graduate Institute of Medicine, Universtiy of Colombo; 2016.

4.Rajapakse T, Christensen H, Cotton S, Griffith K. Nonfatal self-poisoning across age groups, in Sri Lanka. Asian Journal of Psychiatry. 2016;19:79-84.

5.Rethans J, Norcini J, Blackmore D. The relationship between competence and performance: implications for assessing practice performance. Medical Education. 2002;36 (10):901-9.

6.Provincial Director of Health Services Southern. Annual Health Bulletin. In: Health, editor. Galle: Provincial Director of Health Services; 2014.

7.Unni SKE, Mani AJ. Suicidal Ideators In The Psychiatric Facility of A General Hospital - A Psychodemographic Profile. Indian Journal of Psychiatry. 1996;38(2):79-85.

8.National Collaborating Centre for Mental Health. The Short-Term Physical and Psychological Management and Secondary Prevention of Self-Harm in Primary and Secondary Care. Leicester 2004. 